\title{
Modified Wide Tangent Field
}

National Cancer Institute

\section{Source}

National Cancer Institute. Modified Wide Tangent Field. NCI Thesaurus. Code C152053.

A radiation therapy technique used to treat breast malignancies where the treatment fields are wide superiorly to include the internal mammary nodes, but narrow inferiorly to reduce radiation exposure to the heart and lung. 